\title{
BIOMECHANICAL PROPERTIES OF DENTAL IMPLANT WITH ANCHORAGE STRUCTURE IN THE RESTORATION OF UPPER INCISORS MISSING
}

\author{
Fangqiu Ji', Jian Mao ${ }^{2}$, Yufeng Zhou ${ }^{3}$, Chunyu Zhang ${ }^{4}$, Xianshuai Chen ${ }^{5}$, Kehan Feng ${ }^{6}$, \\ Lingfeng $\mathrm{Ye}^{7}$ \\ ${ }^{I}$ School of Mechanical Engineering, Shanghai University of Engineering Science, Shanghai, 201620, China \\ ${ }^{2}$ School of Mechanical Engineering, Shanghai University of Engineering Science, Shanghai, 201620, China \\ ${ }^{3}$ School of Mechanical Engineering, Shanghai University of Engineering Science, Shanghai, 201620, China \\ ${ }^{4}$ Guangzhou Janus Biotechnology Co., Itd. Floor 1, Building A, No.1121 Haibin Rd., Nansha, Guangzhou, 511458, \\ China \\ ${ }^{5}$ Guangzhou Janus Biotechnology Co., Itd. Floor 1, Building A, No.1121 Haibin Rd., Nansha, Guangzhou, 511458, \\ China \\ ${ }^{6}$ Guangzhou Janus Biotechnology Co., Itd. Floor 1, Building A, No.1121 Haibin Rd., Nansha, Guangzhou, 511458, \\ China \\ ${ }^{7}$ Automobile NCO school of Military Transportation College, NO. 1155 Yanshan Road, Yuhui District, Bengbu, Anhui, \\ 233000, China
}

\begin{abstract}
The structure of dental implant is very important to its service life. In order to investigate the effect of the biomechanical properties of anchored dental implant on the osseointegration strength and secondary stability of dental implant, a dental implant model was established based on the three-dimensional structure of human oral cavity, craniomaxillofacial region. Static analysis of dental implant under static load was carried out by using ANSYS finite element analysis software. The results show that the anchorage structure dental implants have better biomechanical compatibility than the traditional standard dental implants, and can improve the strength of the bone-bonding interface. At the same time, the side of the anchorage structure can share the stress of the alveolar bone-implant interface and reduce the risk of unintentional bone-implant damage, so as to ensure the secondary stability of the dental implant, and can better limit the degree of freedom of movement of the dental implant, indirectly promote the restoration of bone-implant injury by reducing the fretting of the dental implant.
\end{abstract}

Keywords: - Anchor fixation structure; Dental implant; Biomechanics; Stability; Finite element analysis ****

\section{INTRODUCTION}

Dental implant prosthesis involves stomatology, mechanical engineering, computer science and other disciplines, which is a multi-disciplinary biomedical engineering problem [1]. Due to the lack of in-depth knowledge of mechanical engineering and the limitation of traditional manufacturing methods, the structure of dental implants on the market is relatively single. At present, the mature dental implant technology uses the shape of the implant is similar to the screw, and the rotary structure is used to achieve the initial fixation of the implant and alveolar bone by thread connection and interference [2]. Such a structure design is easy to manufacture and install, but the human bone structure is biologically active, which is different from the metal structure [3], so this should be considered in the structure design of dental implants.
Due to the limitation of single structure, the current research on the main dental implants has been limited to the torque, screw type, tooth spacing and other parameters related to thread connection [4]. There is a lack of research on the new mechanical structure, and the experimental model of dental implants does not take into account the differences in oral conditions of specific patients [5]. Because the initial stability is the key to ensure the success of the implant [6], many scholars pay attention to the study of the initial stability of the implant, but neglect the influence of the secondary stability on the life of the dental implant. At the beginning of implantation, the mechanical locking effect of interference and torque load is the best when the implant is just implanted; at the second stage, the adaptive self-regulation period of bone tissue structure, the interference and torque load gradually decrease [7]. The mechanical locking effect of the contact interface between the implant and the alveolar bone gradually disappears after the osseointegration of the implant and the alveolar bone, 
and the implant can only be fixed by the osseointegration. However, the bone bond strength of dental implants is not ideal at the beginning of the stress work. Bone bond damage is easy to occur, which will affect the stability of dental implants. Therefore, the two stage stability of dental implants has an effect on the life of dental implants.

In the daily life of patients, large masticatory load and alternating load can cause bone destruction at the bone junction, resulting in a decrease in bone binding effect [8]. When the osseointegration effect falls to the limit, the implant will be loosened, but when the degree of loosening is small, it does not affect the normal progress of bone repair. In the whole process, if the osseointegration can not be repaired in time, it will lead to more and more serious damage and eventually lead to implant loss. According to the statistics of existing medical cases, the majority of implant failure cases are loosening and falling off of implants [9]. Therefore, it is very important to ensure the stability of the implant, that is, to ensure the relative displacement between the implant and the alveolar bone does not occur.

\section{MATERIALS AND METHODS}

\subsection{Structural Design of Dental Implant}

Since the emphasis of this study is on the biomechanical properties of the alveolar bone and the stability of the interface between the implant and the alveolar bone, which does not involve the mechanical properties of the implant itself, it is assumed that the mechanical properties such as the structural strength and stiffness of the dental implant meet the working requirements, and the implant is neglected Effect of body structure on mechanical properties of implant. At the same time, it is assumed that the alveolar bone is fully contacted with the dental implant, that is, the alveolar bone will closely wrap the implant bone segments. In view of the shortcomings of the current dental implant research field, the anchor structure dental implant is proposed. As shown in Fig. 1, it is mainly composed of the main structure and anchorage structure.

In order to study the effect of anchorage structure on implant biomechanical properties and implant stability, three different structural designs, namely triangle structure, square structure and arc structure, were proposed according to the shape of anchorage structure. The width of each individual anchorage structure in the design of intermittent anchorage structure is $1.0 \mathrm{~mm}$, the total length of implant is $12.0 \mathrm{~mm}$, the diameter of the main structure is $4.5 \mathrm{~mm}$, and the diameter of the most peripheral arc of the anchorage structure is $5.5 \mathrm{~mm}$. The height of anchorage structure is designed according to the position of anchorage structure relative to cortical bone. The three heights are set as $9.0 \mathrm{~mm}$, $10.0 \mathrm{~mm}$ and $11.0 \mathrm{~mm}$ respectively. At the same time, two implants with $4.5 \mathrm{~mm}$ diameter and external threads were added for comparison experiment. The screw thread was designed with standard trapezoidal thread. The pitch was 0.5 $\mathrm{mm}$, the tooth angle was 30 degrees, the diameter at the thread was $5.0 \mathrm{~mm}$, the diameter was $4.5 \mathrm{~mm}$, and the length was $12.0 \mathrm{~mm}$. Based on the idea of control variables, the thread design with the same parameters is also used for the anchorage structure of implants.

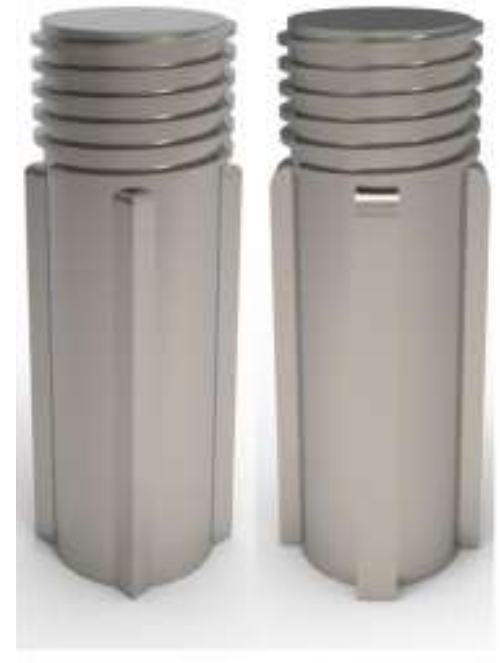

(a)

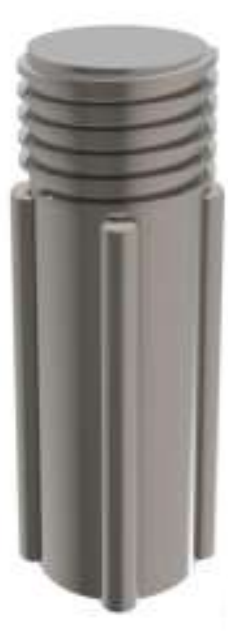

(c)

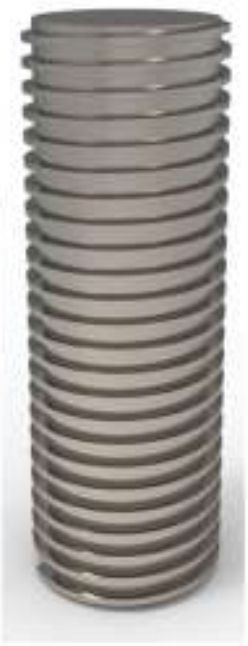

(d)

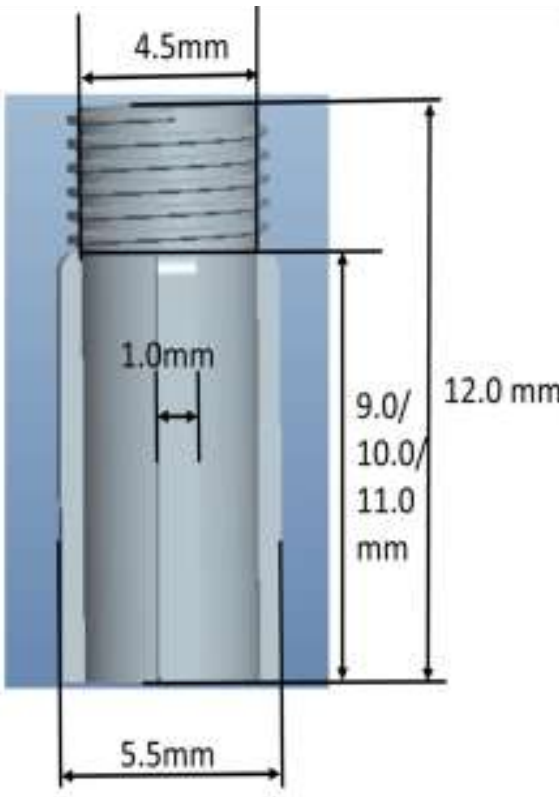

(e)

Fig 1: Sketch map of implant structure and size: (a) triangular anchor fixed structure; (b) square anchor fixed structure; (c) arc anchor fixed structure; (d) standard thread structure; (e) dimension diagram. 


\subsection{Static Load Simplification}

The key to establish the implant model is the determination of the load and the key parameters of the alveolar bone. In order to improve the accuracy of the experimental data, a simulated implant restoration model was established for adult males. Two-dimensional images were obtained by CT scanning of the skull of adult males with missing left incisors during occlusion of upper and lower jaw incisors. A three-dimensional skull model of adult male patients was established by three-dimensional reconstruction function of Mimics software, as shown in Fig. 2(a).

It can be clearly seen that there is a difference in the form of the static load on each tooth in the model, so it is necessary to analyze the load on the implant after the restoration of the missing left upper incisor. Based on the principle of bionics, the implant direction of restored teeth should be similar to the external shape of the denture, especially the crown shape. The missing left upper incisor was restored in situ by Magics software Boolean operation, as shown in Fig. 2(b). The occlusal surface is fitted according to the occlusal points of the upper and lower teeth in the three-dimensional skull model. The formation of occlusal surface is based on people's chewing habits, through long-term wear and squeeze teeth, so that the length of each tooth close to the optimal [10]. The optimal situation should be when the maxillary and maxillary occlusion when the utilization of the masticatory muscle group is the highest, that is, the effect of chewing force on the outside of the occlusal force is close to zero, at this time the direction of chewing force should be perpendicular to the occlusal surface. Based on the human masticatory posture, the upper jaw remains stationary with the skull as the reference, and the mandible is occluded by rotation. However, the rotation axis is not fixed, so there is not only one degree of freedom of rotation, so the forces parallel to the occlusal plane are arbitrary and uncertain. Masticatory muscles play a major role in masticatory movement. Masticatory muscles are used to pull the lower jaw close to the upper jaw. The force acting on the masticatory muscles is approximately perpendicular to the occlusal surface of the upper and lower jaws. Therefore, the component parallel to the occlusal surface is small and can be neglected.

The relative position of the left upper incisors and the lower incisors was extracted from the model, as shown in Fig. 2(c). When the left upper incisor and the lower incisor participate in mastication, the masticatory force is transmitted to the food through the lower incisor and then to the upper incisor, so the force acting on the contact surface between the two teeth. A 3-matic STL software was used to cut the skull based on the center line position of the left incisor contact surface, then projected the occlusal posture of the left upper and lower incisors onto the incisor surface, and then connected the two end points of the upper incisor projection surface to get a straight line, which was defined as the tooth axis, as shown in Fig. 2(c). The movement trend of the lower incisor decides the direction of the force. It is known from the above analysis that the movement trend of the lower jaw is close to the upper jaw, and when the upper and lower jaws occlude, the direction of the force is perpendicular to the occlusal surface. Since the lower incisor and the mandible are integral, the occlusal force on the upper incisor is perpendicular to the occlusal surface, and the angle between the occlusal force and the tooth axis is 31.6 degrees. Because the incisor plays a small role in the actual mastication process, so it suffers less static load, so $100 \mathrm{~N}$ is selected as a single tooth occlusal force [11]. According to the extraction socket and tooth shape, the implant direction and the shape of the implant crown were determined. Finally, the implant structure was obtained by combining the crown with the implant as shown in Fig. 2(d), in which the central line of the implant coincided with the axis of the left upper incisor.

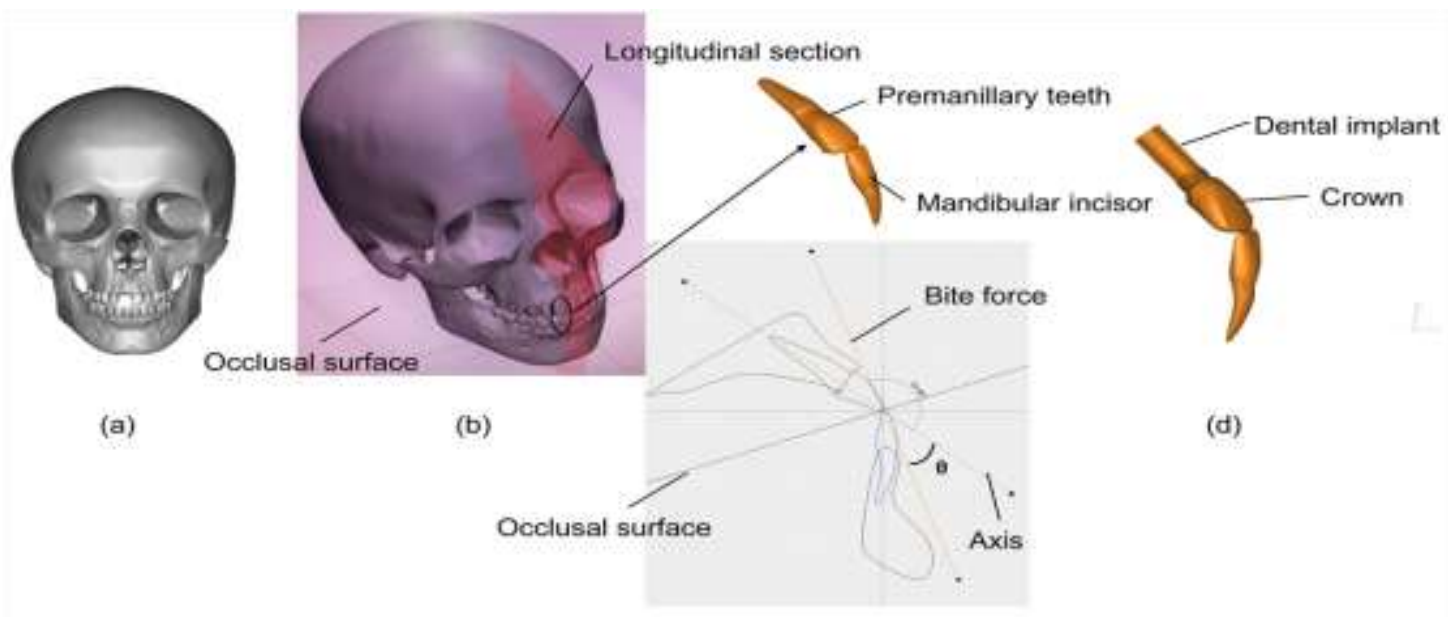

(c)

Fig 2: (a) a three-dimensional model of the skull; (b) a three-dimensional model of the left upper incisor repaired by a Boolean operation; (c) the projection of the relative position of the left upper incisor and the lower incisor on the cutting surface; (d) the structure of the dental crown and the implant to the implant. 


\subsection{Alveolar Bone Model Simplification}

The alveolar bone is composed of cortical bone and cancellous bone. It differs from ordinary materials in that it has biological activity. The essence of biological activity is that it can self repair, but this process takes time. Therefore, when the static load is applied, it can be regarded as ordinary plastic material, so the parameters of alveolar bone can be set according to the ordinary plastic material. The cortical bone thickness of the missing teeth was distributed at $1.5 \mathrm{~mm}$ to $2 \mathrm{~mm}$ by CBCT. In order to facilitate the ANSYS finite element simulation, the alveolar bone model is simplified without affecting the accuracy of the model. One of the simplified procedures is described in detail as shown in Fig. 3. Firstly, the implant was installed in the missing dentition by Boolean operation of Magics software, and then the alveolar bone was cut out to support the implant load. Finally, the alveolar bone model was obtained by restoration. Because the cortical bone in the human skeleton surrounds the cancellous bone in the outermost layer, the model was set to surround the cancellous bone with the cortical bone. The final simplified force model is shown in Fig. 3(d), which consists of cortical bone, cancellous bone and dental implant. The static load is $100 \mathrm{~N}$, the direction of action is 31.6 degrees with the axis, and the force action area is near the highest point of the tooth crown.

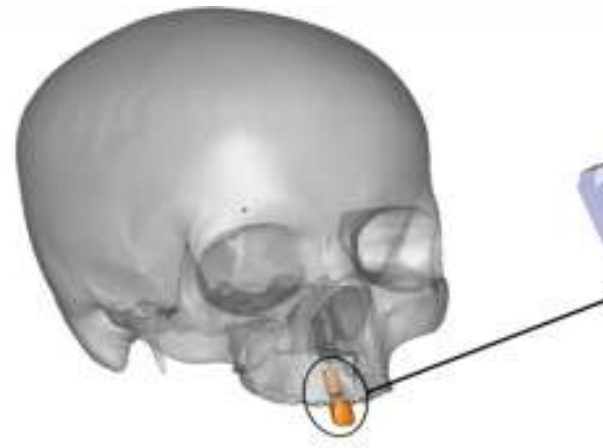

(a) (b)

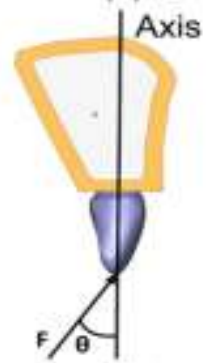

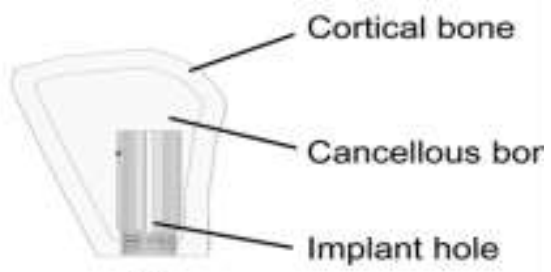

(c)

(d)

Fig 3: Simplification of experimental model: (a) the effect map of dental implants after implant; (b) truncated alveolar bone unit with dental implants; (c) simplified model of alveolar bone; (d) an experimental model of force.

\subsection{Finite Element Analysis}

Assuming that both cortical and cancellous bones meet the isotropic requirements, the relevant parameters are set according to A. Dorogoy's model [12] as shown in Table 1. The implants and crowns designed in this paper are printed in 3D with pure titanium powder based on SLM technology, so the parameters of implants and crowns are shown in
Table 1. At the same time, it is assumed that the implant has a good osseointegration with the alveolar bone, so the contact mode between the alveolar bone and the implant is set as binding. The alveolar bone model and the implant model were meshed by 3-matic STL, and the fine mesh was made according to the contact position between the alveolar bone and the implant.

Table 1: Young's modulus, Poisson's ratio and Density for the test materials: CP Ti; cancellous and cortical bone.

\begin{tabular}{|l|l|l|l|}
\hline Material & Young's modulus $(\mathrm{E}, \mathrm{GPa})$ & Poisson's ratio $(\mathrm{V})$ & Density $(\rho, \mathrm{kg} / \mathrm{m} 3)$ \\
\hline Cortical bone & 18 & 0.35 & 1900 \\
\hline Cancellous bone & 0.7 & 0.35 & 1000 \\
\hline CP Ti & 110 & 0.34 & 4510 \\
\hline
\end{tabular}




\section{EXPERIMENT}

The experimental model with well-meshed body is imported into ANSYS finite element analysis software, and the stress analysis diagrams of different structures are obtained. Fig. 4 is the equivalent stress diagram of alveolar bone after implantation of standard structure dental implants. The maximum equivalent stress appears on the outer surface of cortical bone, which is $27.712 \mathrm{MPa}$. At the same time, there is stress concentration at the maximum equivalent stress of cortical bone, the equivalent stress is about 15-24 MPa, and the stress range is about $3 \mathrm{~mm}$ below the cortical bone apex. Stress concentration also exists in cancellous bone. The stress range is less than $4 \mathrm{~mm}$ from the top to the bottom along the implant socket, and the stress is about 0.5-1.1 MPa. The maximum stress was $1.3549 \mathrm{MPa}$, which appeared in the first thread grooves on the top of cancellous bone.

Fig. 5 is the equivalent stress diagram of the alveolar bone after implanting three kinds of dental implants with $9 \mathrm{~mm}$ long anchorage structure. The distribution of the equivalent stress concentration area on the cortical bone of the three models is similar, and the stress action range is about $3 \mathrm{~mm}$ below the cortical bone apex, and the equivalent stress in the concentrated area is about 15-24 MPa. However, the stress distribution on cancellous bone is quite different. The maximum equivalent stress of alveolar bone of dental implants with triangular anchorage structure appears at the top of cancellous bone. The stress concentration area extends about $7.5 \mathrm{~mm}$ along the maximum equivalent stress, and the equivalent stress is about 0.5-1.0 $\mathrm{MPa}$. The maximum equivalent stress of the alveolar bone of the dental implant with square anchorage structure appears at the top of the square groove which contacts with the anchorage structure. The stress concentration exists in the square groove near the maximum equivalent stress. The concentration area is about $2.0-7.5 \mathrm{~mm}$ below the top of the implant socket, and the equivalent stress is about 0.8-1.8 $\mathrm{MPa}$. The maximum equivalent stress of the alveolar bone of dental implants with arc anchorage structure appears at the top of cancellous bone, but the stress concentration is similar to that of the alveolar bone of dental implants with square anchorage structure. The equivalent stress is about 0.9-1.9 MPa.

Fig. 6 is the equivalent stress diagram of the alveolar bone after implantation of three kinds of implants with $10 \mathrm{~mm}$ long anchorage structure. The distribution of the equivalent stress concentration area on the cortical bone of the three models is similar, and the stress action range is about $3 \mathrm{~mm}$ below the cortical bone apex, and the equivalent stress in the concentrated area is about 15-24 MPa. . However, the stress distribution on cancellous bone is quite different. The maximum equivalent stress of the alveolar bone of the dental implant with triangular anchorage structure appears at the top of the anchorage structure. The stress concentration area is within $5 \mathrm{~mm}$ from the maximum equivalent stress position, and the equivalent stress is about 0.7-1.2 MPa. The maximum equivalent stress of the alveolar bone of the dental implant with square anchorage structure also appears at the top of the anchorage structure. The stress concentration area is within $5 \mathrm{~mm}$ from the maximum equivalent stress position down the anchorage structure, and the equivalent stress is about 1.0-2.0 MPa. The maximum equivalent stress of the alveolar bone of dental implants with arc-shaped anchorage structure appears on the screw thread at the top of the implant socket. The stress concentration area is within $5 \mathrm{~mm}$ from the maximum equivalent stress position downward along the anchorage structure, and the equivalent stress is about $0.5-1.0 \mathrm{MPa}$.

Fig. 7 is the equivalent stress diagram of the alveolar bone after implantation of three kinds of implants with $11 \mathrm{~mm}$ long anchorage structure. The distribution of the equivalent stress concentration area on the cortical bone of the three models is similar, and the stress diffuses from the top position of the anchorage structure to the surrounding area. The range of action is about $3 \mathrm{~mm}$ below the top of the cortical bone. The equivalent stress in the concentrated area is about 18 28 MPa. However, there are differences in the location of stress concentration in three situations. The distribution of the equivalent stress concentration area and the location of the maximum stress in the cancellous bone of the three models were similar. The stress concentration area was within $5 \mathrm{~mm}$ from the top of the implant socket to the bottom. The equivalent stress of the alveolar bone with triangular anchorage was about 0.8-1.4 $\mathrm{MPa}$, and the alveolar bone with square anchorage structure was implanted. The equivalent stress is about 1.0-1.9 $\mathrm{MPa}$, and the equivalent stress of alveolar bone implanted with arc anchor is about 1.5-2.3 $\mathrm{MPa}$. 


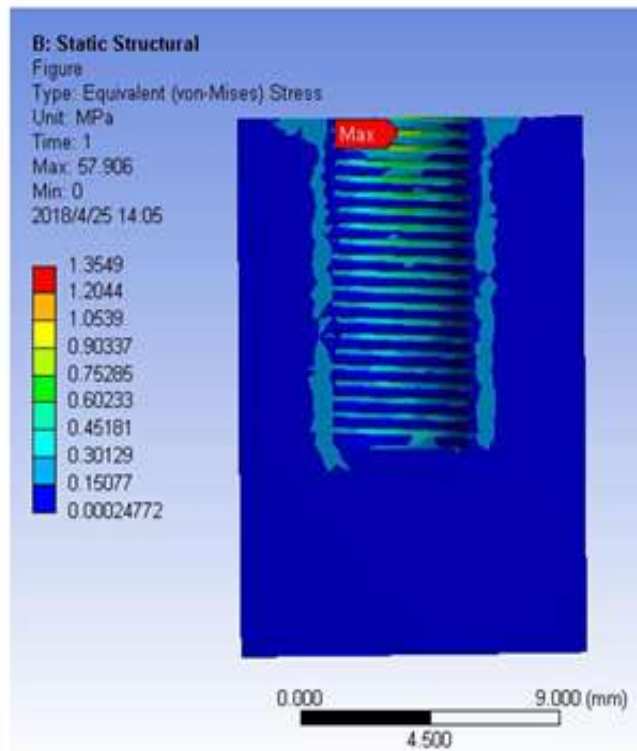

(a)

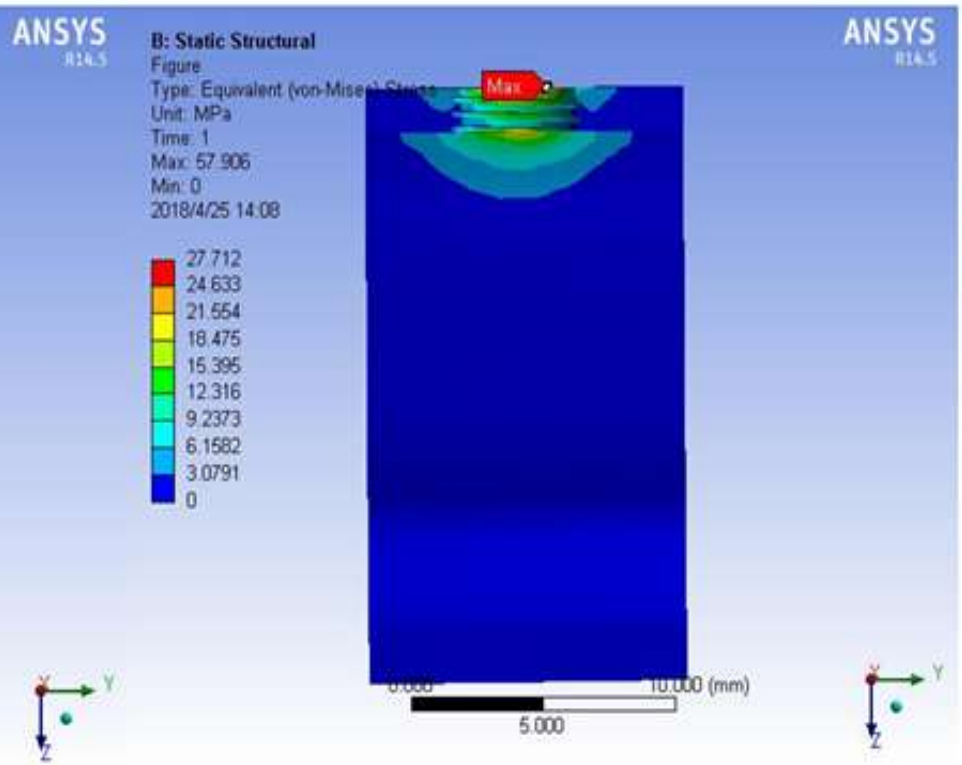

(b)

Fig 4: The equivalent stress diagram of alveolar bone implanted with standard screw implant: (a) cancellous bone; (b) cortical bone.

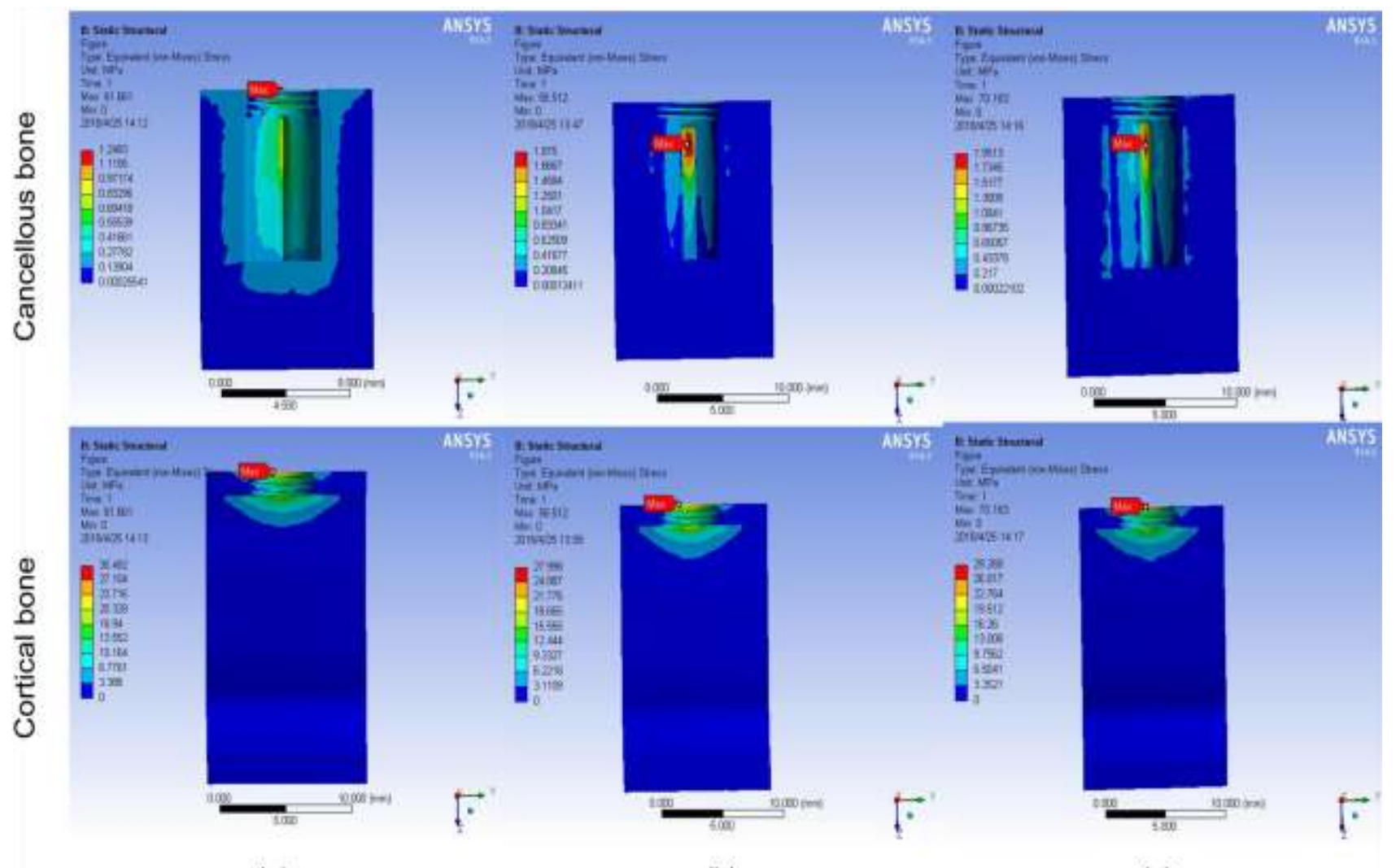

(a)

(b)

(c)

Fig 5: The equivalent stress stripe of the alveolar bone with a $9 \mathrm{~mm}$ long anchorage fixed dental implant was planted: (a) a triangular anchor fixed dental implant; (b) a square anchorage fixed dental implant; (c) a curved anchorage implant. 


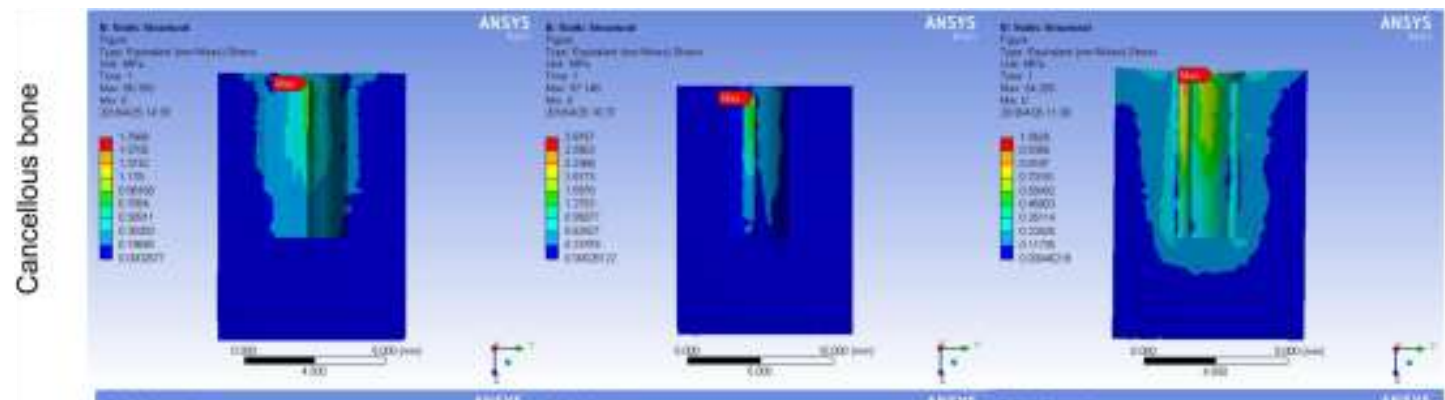

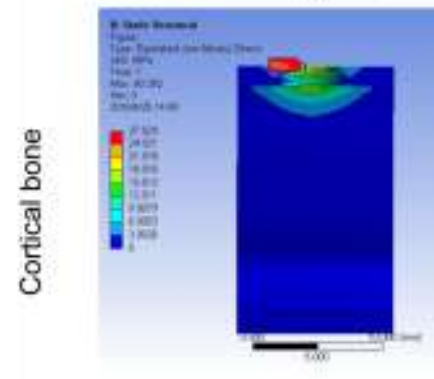

(a)

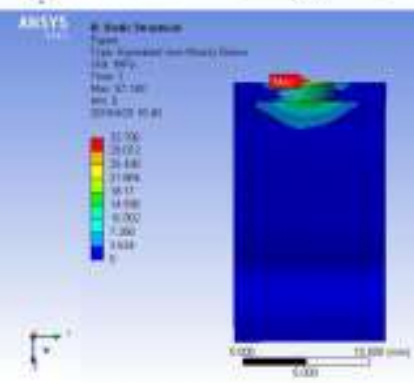

(b)

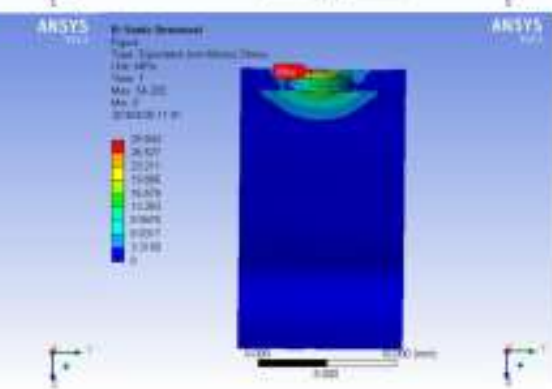

(c)

Fig 6: The equivalent stress stripe of the alveolar bone with a $10 \mathrm{~mm}$ long anchorage fixed dental implant was planted: (a) a triangular anchor fixed dental implant; (b) a square anchorage fixed dental implant; (c) a curved anchorage implant.
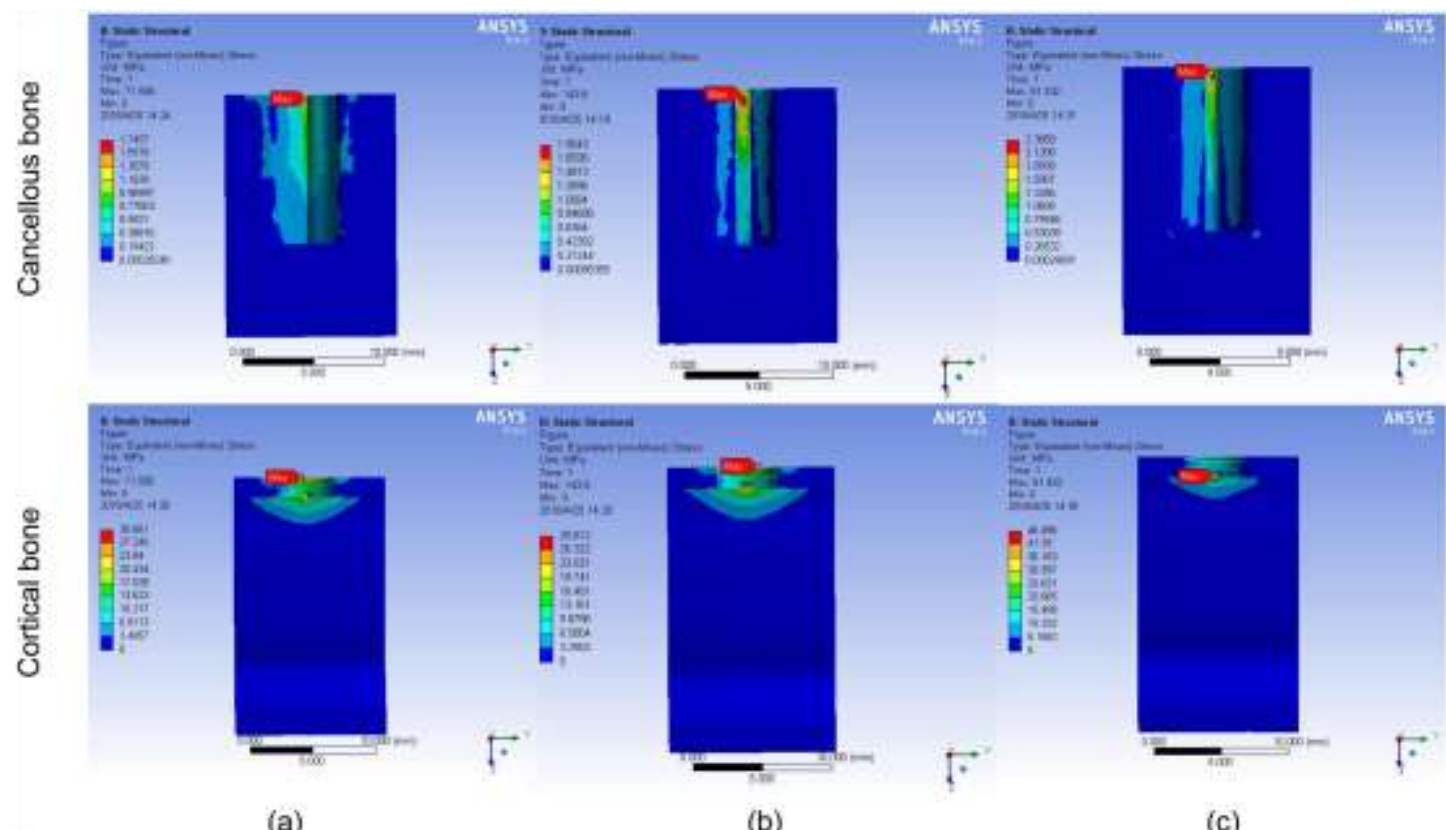

(a)

(b)

(c)

Fig 7: The equivalent stress stripe of the alveolar bone with a $11 \mathrm{~mm}$ long anchorage fixed dental implant was planted: (a) a triangular anchor fixed dental implant; (b) a square anchorage fixed dental implant; (c) a curved anchorage implant.

\section{DISCUSSION}

In this paper, three types of dental implants are proposed, each of which contains three size parameters and contains nine types of experimental models. The stress distribution on the cortical bone showed that only the alveolar bone implanted with $11 \mathrm{~mm}$ long anchor was significantly different from that of the alveolar bone implanted with standard thread dental implant. This is because only $11 \mathrm{~mm}$ long anchorage structure has contact with cortical bone, the rest of the structure contact with cortical bone are threaded segments. By comparing the equivalent stress peaks of the cortical bone in Fig. 8, it can be seen that the stress peak value of the cortical bone implanted with $11 \mathrm{~mm}$ long arc anchor structure is $46.496 \mathrm{MPa}$. Bone threshold theory is currently widely used in bone biomechanical evaluation criteria [13]. The theory proposes that the strain range of bone under normal functional state is 50-3000 micro-strain, which is equivalent to the load applied on bone is 1-60 MPa. Therefore, the stress of cortical bone in nine types of experimental models is within the normal range. According 
to Frost's hypothesis [14], osteocytes respond to local bone deformation caused by mechanical pressure. By slowly increasing strain and mildly overloading, bones compensate for this deformation by forming more bones. And Wolff's Law [15] holds that bones have functional adaptability, and they can also adapt to load changes in their own structure. Therefore, in normal functional state, the greater the stress, the more conducive to the growth of bone [16], thus increasing the strength of bone bonding. Therefore, compared with other experimental models, the implants with $11 \mathrm{~mm}$ long curved anchorage structure have better biomechanical compatibility with cortical bone.

The equivalent stress distribution on the cancellous bone model with anchored implants is obviously different from that of the cancellous bone model with standard threaded implants. The stress area of cancellous bone of dental implants with $9 \mathrm{~mm}$ long anchorage structure was the largest, and that of cancellous bone with $10 \mathrm{~mm}$ and $11 \mathrm{~mm}$ long anchorage structure was the second but also greater than that of cancellous bone of dental implants with standard thread structure. Among the nine types of implants, the equivalent stress of $11 \mathrm{~mm}$ anchorage structure on cancellous bone is the largest. According to the peak value of equivalent stress of cancellous bone, the peak value of equivalent stress of cancellous bone between $10 \mathrm{~mm}$ Rectangular anchorage structure and $11 \mathrm{~mm}$ arc anchorage structure is relatively large. Based on the above Wolff's law, $11 \mathrm{~mm}$ dental implants with curved anchorage structure have the best biomechanical compatibility with cancellous bone.

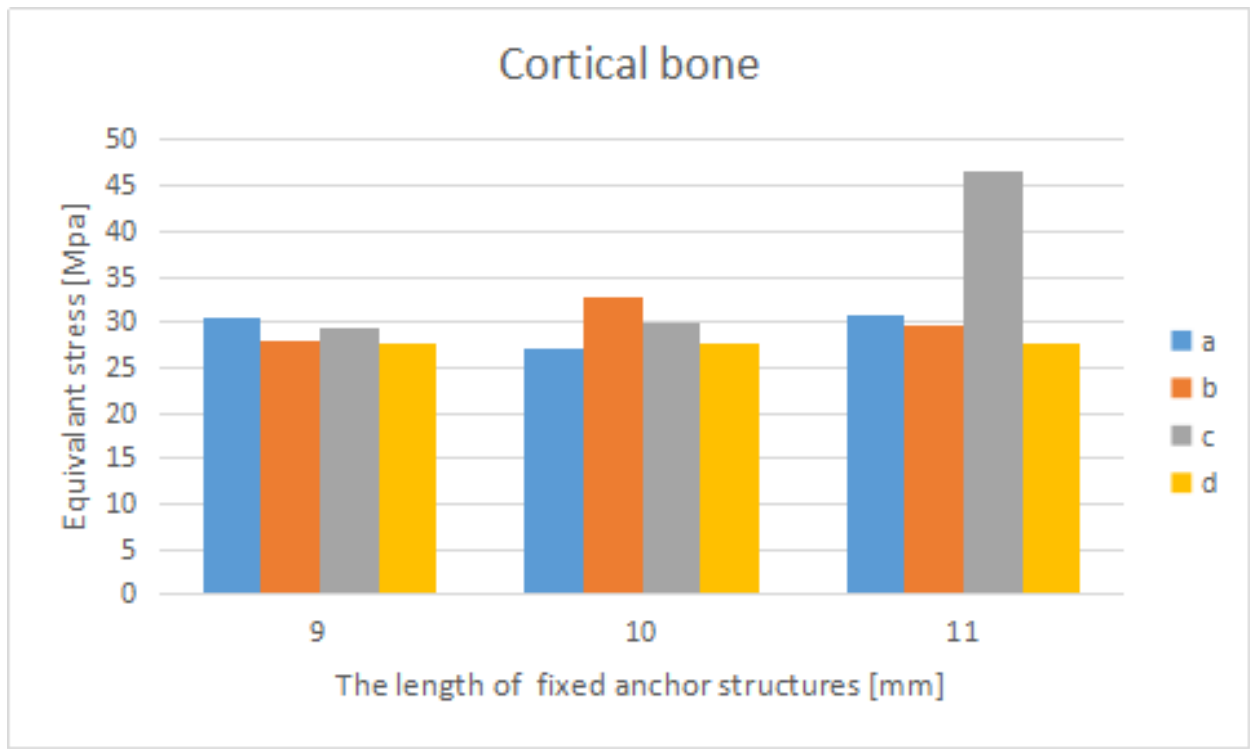

Fig 8: Comparison of the stress peak value of the cortical bone: (a) a triangular anchor fixed dental implant; (b) a square anchor fixed dental implant; (c) a curved anchorage implant; (d) a standard threaded dental implant.

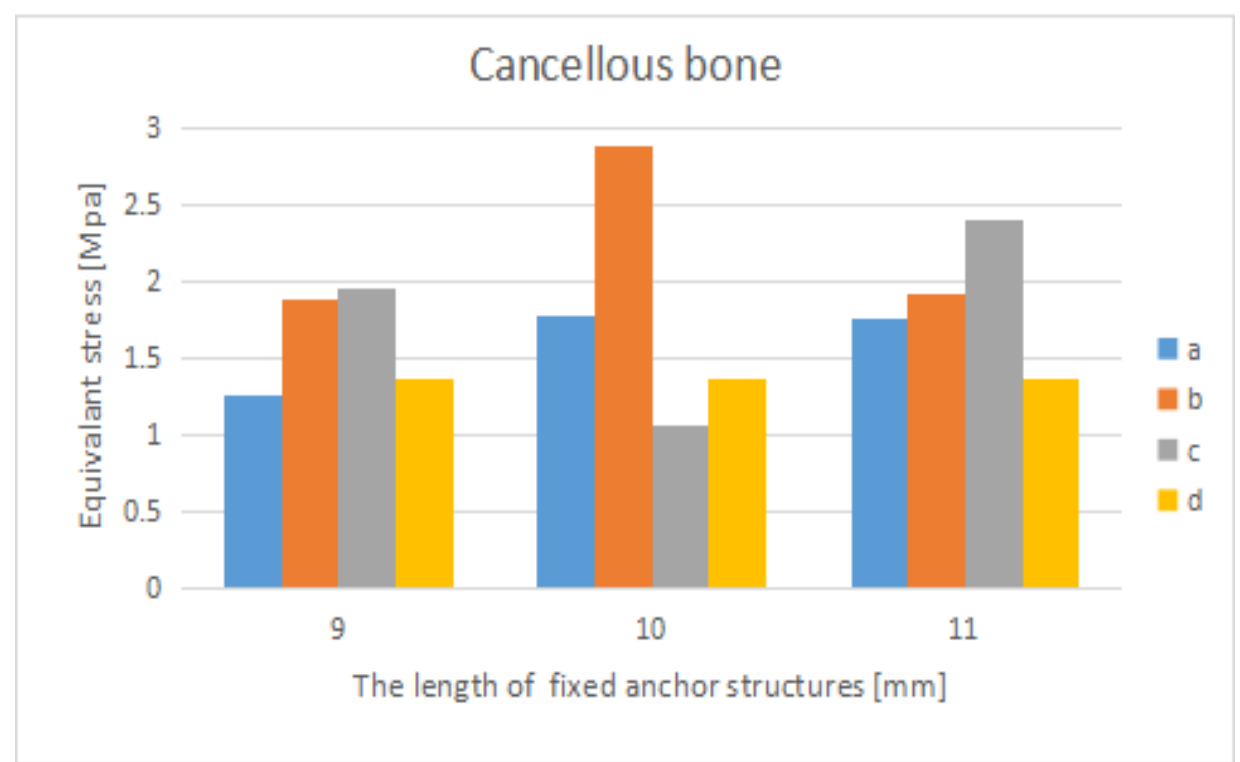

Fig 9: Comparison of the stress peak value of the cancellous bone: (a) a triangular anchor fixed dental implant; (b) a square anchor fixed dental implant; (c) a curved anchorage implant; (d) a standard threaded dental implant. 
In the process of osseointegration, better biocompatibility of stress can promote the proliferation of osteocytes, and thus faster formation of osseointegration [13]. After the formation of osseointegration, continuous stress stimulation can further strengthen the strength of osseointegration and prevent alveolar bone resorption. The stability of dental implant can be defined as the tendency of displacement relative to alveolar bone under the influence of many factors. Considering the alveolar bone as an ordinary elastic-plastic material, the movement trend of the alveolar bone in terms of the degree of freedom of the implant restrained by the alveolar bone is only rotation and downward sliding, as shown in Fig. 10. After full osseointegration of implant and alveolar bone, bone remodeling was basically completed, and the mechanical locking function of implant and alveolar bone disappeared. Therefore, the mechanical locking of threads is no longer conducive to the stability of the implant. However, the lateral contact of the anchorage structure with the alveolar bone can restrict the movement of the implant when it is subjected to torque and tension. Thus, the stress on the alveolar bone-implant interface is shared, and the risk of fracture of the alveolar bone-implant interface is reduced, so the secondary stability of the dental implant is improved. In the normal operation of dental implants, periodontal diseases or mechanical injuries may lead to the breakage of bone bonding, resulting in the reduction of bone bonding [17]. In this case, the anchorage structure can reduce the risk of displacement by restricting the degree of freedom of the implant movement on its side, thus compensating for the osseointegration and ensuring the stability of the implant. Just as osseointegration occurs, the osseointegration injury has a chance to recover when the implant is stable [18]. Therefore anchorage structure can indirectly promote the recovery of osseointegration injury.

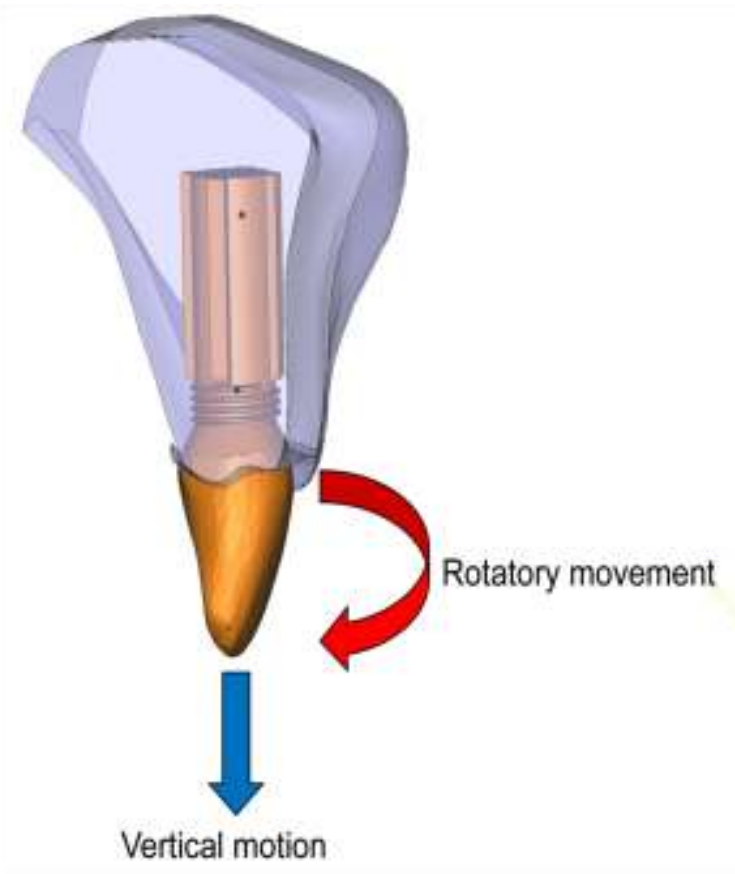

Fig 10: Tendency of implant displacement relative to alveolar bone

\section{CONCLUSION}

In this paper, an anchor-fixed structure dental implant is proposed, which can accelerate the process of osseointegration and enhance the ability of osseointegration compared with the traditional standard threaded structure dental implant at the early stage of implantation. The stability of the implant can be improved by limiting the degree of freedom of the implant by anchorage structure in the case of broken bone bond. According to the results of finite element analysis, $11 \mathrm{~mm}$ long arc anchor fixed structure dental implants have the best biomechanical compatibility compared with other anchor fixed structure dental implants in this paper.

\section{ACKNOWLEDGEMENTS}

This work is supported by Science and Technology Planning Project of Guangdong province, China (No.2017B090901039); Science \& Technology Plan Project of Guangzhou (201710010193); the Science and Technology Program of Nansha District, Guangzhou (Application Technology Research, No. 2016GG020).

\section{REFERENCE}

[1] Michael S. Block. Dental Implants: The Last 100 Years [J]. Journal of Oral and Maxillofacial Surgery. 2018, 76(1): 11-26.

[2] Lin Ye. Current dental implant design and its clinical importance [J]. West China Journal of Stomtology. 2017, 35(01): 18-28.

[3] Youngjune Jang, Hyoung-Taek Hong, Heoung-Jae Chun. Influence of Dentoalveolar Ankylosis on the Biomechanical Response of a Single-rooted Tooth and Surrounding Alveolar Bone: A 3-dimensional Finite Element Analysis [J]. Journal of Endodontics. 2016, 42(11): 1687-1692.

[4] Zembic, S. Kim, M. Zwahlen. Systematic review of the survival rate and incidence of biologic, technical,and esthetic complications of single implant abutments supporting fixed prostheses [J]. Int J Oral Maxillofac Implants. 2014, 29 (Supp): 99-116.

[5] Song HF, Li Q, Ma L, et al. Three Dimensional Finite Element Analysis of the Stress Distribution of Mandible with the Effects of Implant Depths and Force Directions [J]. Beijing Biomedical Engineering. 2009, 28 (6): 573-576.

[6] Jui-Ting Hsu, Yen-Wen Shen, Chih-Wei Kuo. Impacts of 3D bone-to- implant contact and implant diameter on primary stability of dental implant [J]. Journal of the Formosan Medical Association. 2017, 116(8): 582-590.

[7] Jason A. Griggs. Dental Implants [J]. Dental Clinics of North America. 2017, 61(4): 857-871.

[8] F. Wang, Z. Zhang, A. Monje. Intermediate long-term clinical performance of dental implants placed in sites with a previous early implant failure: a retrospective analysis [J]. Clin Oral Implants Res. 2015, 26: 1443-1449. 
[9] Jason A. Griggs, 2017. Dental Implants [J]. Dental Clinics of North America. 61(4), 857-871.

[10] Thomas R. Morneburg, Peter A. Pröschel. Measurement of Masticatory Forces and Implant Loads: A Methodologic Clinical Study [J]. International Journal of Prosthodontics. 2002 , 15 (1): 20-27.

[11] J.R. Sarot, C.M. Contar, A.C. Cruzi. Evaluation of the stress distribution in CFR-PEEK dental implants by the three-dimensional finite element method [J]. Mater. Sci. Mater. Med.2010, 21: 2079-2085.

[12] Dorogoy, D. Rittel, K. Shemtov-Yona. Modeling dental implant insertion [J]. Journal of the Mechanical Behavior of Biomedical Materials. 2017, 68: 42-50.

[13] Quek CE, Tan KB, Nicholls JI. Load fatigue performance of a single-tooth implant abutment system: effect of diameter [J]. The International journal of oral \& maxillofacial implants. 2006, 21: 929-936.

[14] Frost, H.M. Perspectives: bone's mechanical usage windows [J]. Bone and Mineral 1992(19): 257-271.

[15] Allum SR, Tomlinson RA, Joshi R. The impact of loads on standard diameter, small diameter and mini implants: a comparative laboratory study [J]. Clin Oral Implants Res. 2008, 19: 553-559.

[16] Macedo, J.P., Pereira. Morse taper dental implants and platform switching: The new paradigm in oral implantology [J]. Eur. J. Dent. 2016, 10(1): 148-154.

[17] C.-C. Lee, S.-C. Lin, M.-J. Kang. Effects of implant threads on the contact area and stress distribution of marginal bone [J]. J. Dent. Sci. 2010, 5: 156-165.

[18] Bahat O. Treatment planning and placement of implants in the posterior maxillae: report of 732 consecutive Nobelpharma implants [J]. Int J Oral Maxillofac Implants.1993, 8(2): 151-161. 